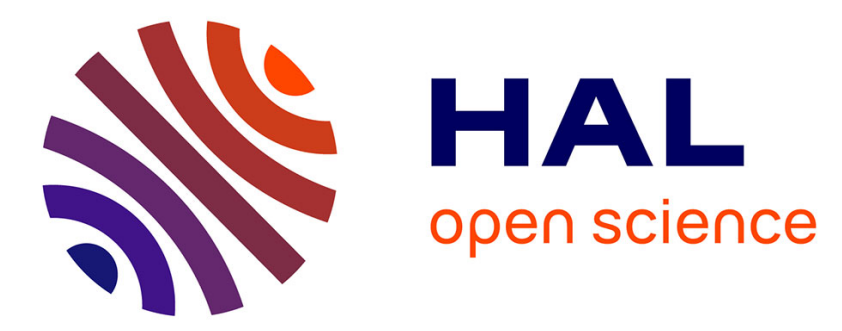

\title{
Des biens communs aux inconnus communs : initier un processus collectif de conception pour la gestion durable d'un agro-écosystème
}

Elsa Berthet, Blanche Segrestin, Benoit Weil

\section{- To cite this version:}

Elsa Berthet, Blanche Segrestin, Benoit Weil. Des biens communs aux inconnus communs: initier un processus collectif de conception pour la gestion durable d'un agro-écosystème. RIODD 2016, Jul 2016, Saint-Étienne, France. hal-01349984

\section{HAL Id: hal-01349984 \\ https://hal.science/hal-01349984}

Submitted on 29 Jul 2016

HAL is a multi-disciplinary open access archive for the deposit and dissemination of scientific research documents, whether they are published or not. The documents may come from teaching and research institutions in France or abroad, or from public or private research centers.
L'archive ouverte pluridisciplinaire HAL, est destinée au dépôt et à la diffusion de documents scientifiques de niveau recherche, publiés ou non, émanant des établissements d'enseignement et de recherche français ou étrangers, des laboratoires publics ou privés. 


\title{
Des biens communs aux inconnus communs : initier un processus collectif de conception pour la gestion durable d'un agro-écosystème
}

\author{
EIsa BERTHET \\ Dr., Université McGill et INRA \\ Sustainable Futures Research Laboratory \\ Département des Sciences des Ressources Naturelles \\ 21,111 Lakeshore, H9X3V9 Sainte-Anne-de-Bellevue, Québec, Canada \\ elsa.berthet@agroparistech.fr
}

\author{
Blanche SEGRESTIN \\ Prof., Mines Paristech \\ Centre de gestion scientifique \\ 60 Boulevard Saint-Michel, 75006 Paris, France \\ blanche.segrestin@mines-paristech.fr
}

\section{Benoit WEIL}

Prof., Mines Paristech

Centre de gestion scientifique

60 Boulevard Saint-Michel, 75006 Paris, France

benoit.weil@mines-paristech.fr

\section{Résumé}

La dégradation des écosystèmes est souvent interprétée comme une problématique de surexploitation de ressources communes. Les écosystèmes cultivés semblent cependant poser de nouvelles questions de gouvernance, car il n'existe ni autorité de gestion, ni « ressources communes » dont chacun percevrait la nécessité pour autoriser une forme de gouvernance locale. Nous présentons un cas empirique dans lequel des acteurs ont tenté de concevoir collectivement un agro-écosystème durable à partir de connaissances récentes en écologie. Nous montrons que les acteurs ont identifié un " inconnu commun » leur permettant d'ouvrir un espace pour l'exploration collective. Cette recherche contribue à la littérature visant à « dénaturaliser » les biens communs, et met en évidence des enjeux de gestion encore largement sous-estimés dans les approches classiques des biens communs.

Mots-clés : Agro-écosystème ; gouvernance ; biens communs ; inconnu commun ; conception collective

Key-words: Agroecosystem ; governance ; commons ; common unknown ; collective design 


\section{Des biens communs aux inconnus communs : initier un processus collectif de conception pour la gestion durable d'un agro-écosystème}

\section{Résumé}

La dégradation des écosystèmes est souvent interprétée comme une problématique de surexploitation de ressources communes. Les écosystèmes cultivés semblent cependant poser de nouvelles questions de gouvernance, car il n'existe ni autorité de gestion, ni « ressources communes » dont chacun percevrait la nécessité pour autoriser une forme de gouvernance locale. Nous présentons un cas empirique dans lequel des acteurs ont tenté de concevoir collectivement un agro-écosystème durable à partir de connaissances récentes en écologie. Nous montrons que les acteurs ont identifié un " inconnu commun » leur permettant d'ouvrir un espace pour l'exploration collective. Cette recherche contribue à la littérature visant à "dénaturaliser » les biens communs, et met en évidence des enjeux de gestion encore largement sous-estimés dans les approches classiques des biens communs.

\section{Introduction}

Depuis les travaux fondateurs d’Ostrom, la problématique de la durabilité de nombreux écosystèmes anthropisés, tels que les lacs, les forêts et les pâturages, est interprétée comme étant liée à la gestion de biens communs. L’accent est alors mis sur l’analyse de la capacité des acteurs à s'auto-organiser pour assurer une gestion durable des ressources communes. Cependant, les agro-écosystèmes, ou écosystèmes cultivés, qui produisent une diversité de ressources pour lesquelles les acteurs ont des intérêts et logiques d'action souvent divergents, posent des questions nouvelles en termes de gouvernance : il n'existe pas d'autorité de gestion, ni de ressources communes dont chacun percevrait la nécessité pour autoriser une forme de gouvernance locale. Comment appréhender une telle diversité de ressources et de valeurs qui y sont associées ? Comment recréer des liens entre des acteurs aussi hétérogènes que les agriculteurs, les naturalistes, les consommateurs, etc. de façon à ce qu'ils initient une forme d'auto-organisation ? Cet article s’interroge sur la possibilité de mobiliser le cadre 
d'analyse d'Ostrom pour traiter la question de la gouvernance des agro-écosystèmes, et propose de l'élargir à une classe d'actions plus large que la préservation de ressources communes.

Tout d'abord, l'article rappelle brièvement les principaux apports d'Ostrom sur la gouvernance des biens communs. Puis il montre, à partir de la description d'un cas emblématique des travaux d'Ostrom, qu'en stylisant les systèmes de ressources communes à partir des différents cas étudiés, l'auteur a formulé des hypothèses qui circonscrivent le domaine d'application de son cadre d'analyse. Afin d'analyser précisément les enjeux de gouvernance soulevés par les agro-écosystèmes, l'article s’appuie sur un cas empirique récent. Il montre que, contrairement à ce que l'on pourrait croire initialement, l'agro-écosystème ne peut être considéré comme un système de ressources communes tel que modélisé par Ostrom. Dans le cas étudié, une démarche de conception innovante a été expérimentée dans le cadre d'un projet porté par une coopérative agricole et un centre de recherche en écologie, afin d'identifier ce qui pouvait être géré en commun. Nous analysons et interprétons ce cas en mobilisant la théorie C-K (Concept-Knowledge) de la conception innovante (Hatchuel and Weil 2009). L'analyse du cas met en évidence que les acteurs mobilisent des connaissances en écologie pour identifier un « inconnu commun » (Le Masson et Weil 2014), qui constitue le point de départ d'un processus d'exploration de ce qui peut être fait collectivement pour améliorer la durabilité de l'agro-écosystème. L’identification et l'exploration d’un inconnu commun semblent constituer une piste intéressante pour générer une action collective alors qu'il n’y a pas de bien commun a priori. Cependant, un tel processus soulève des enjeux de gestion encore peu identifiés aujourd’hui dans la littérature sur les biens communs. 


\section{1 : Le paradigme du stock à préserver dans la littérature sur les biens communs}

\section{1 : Le marché ou la régulation publique comme solutions à la tragédie des communs}

Les économistes définissent les biens communs par les caractéristiques suivantes : la nonexclusivité, i.e., il est très difficile, voire impossible, d’exclure des bénéficiaires de ce bien, par différence avec les biens privés ; et la rivalité, i.e., le bien peut être dégradé lors de son utilisation, par différence avec les biens publics purs. Comme le souligne Billaudot (2013), la question posée est de savoir quelle forme d'institution prend la coordination entre individus pour disposer de biens. Or, cette définition des biens communs ne concerne que les problématiques liées à l'usage du bien et non celles liées à sa production : la question de la nécessité ou non d’une production collective par exemple n’est pas mentionnée. Labatut (2009) qualifie la perspective économique néo-classique des biens communs, de « naturaliste », car les biens sont considérés comme préexistants et identifiés par les acteurs, et d' « utilitariste », car les acteurs sont vus comme cherchant à maximiser leur propre utilité. En d'autres termes, les biens et les comportements des acteurs sont considérés comme donnés.

Selon la perspective économiste, les biens communs, comme les externalités, peuvent entraîner des défaillances de marché, et peuvent susciter des comportements de passager clandestin ${ }^{1}$. L’identification de ce problème a donné lieu à un certain nombre de théories influentes dans la sphère scientifique comme politique dans les années 1960 à 1980, notamment le dilemme des prisonniers, la logique de l'action collective (Olson 1965) et la tragédie des communs (Hardin 1968). Ces théories prédisent que les biens communs sont

\footnotetext{
${ }^{1}$ Acteur qui obtient et profite d'un avantage obtenu ou créé par un groupe de personnes ou par la collectivité, que ce soit une situation favorable, un bien ou un service, sans y avoir investi autant d'efforts que les membres de ce groupe, ou sans acquitter leur juste quote-part ou le droit d'usage prévu.
} 
inévitablement voués à une surexploitation ou une dégradation, et proposent deux solutions principales pour y remédier : d'une part la privatisation des biens et leur gestion par le marché, d'autre part la régulation publique (taxation, réglementation de l'accès au bien, etc.) ces deux solutions n'étant pas exclusives l'une de l'autre, les instruments de marché étant généralement l'expression des pouvoirs publics.

\subsection{Une troisième voie mise en avant par Ostrom : l'auto-organisation}

Ostrom s'est intéressée à différents types de biens communs, qu’il s'agisse de ressources naturelles (nappes phréatiques, pêcheries, forêts, pâturages), ou de biens d'origine anthropique (systèmes d'irrigation, connaissances). Ostrom qualifie ces biens de ressources communes (Common Pool Resources) qu'elle définit comme un « système de ressources naturelles ou d'origine humaine suffisamment grand pour qu'il soit coûteux (mais pas impossible) d'en exclure des bénéficiaires potentiels »(Ostrom, 1990, p. 30, notre traduction). Elle distingue le système de ressources, qui nécessite une gestion commune en raison d'un certain nombre de caractéristiques spécifiques, et les unités de ressources, qui, elles, sont appropriables par des individus. Elle considère les ressources comme un stock et les unités collectées comme des flux. Sa perspective peut donc être qualifiée de naturaliste (Labatut 2009).

En revanche, contrairement aux économistes néo-classiques, Ostrom rejette l’idée que les comportements des acteurs sont donnés, et s'intéresse aux conditions dans lesquelles ils peuvent changer. Selon elle, les situations d'action influencent le comportement des acteurs (Ostrom 1990); or ces situations d'action peuvent être modifiées par des institutions (e.g., règles, normes et valeurs) que les acteurs sont capables d'élaborer eux-mêmes. Ceci l'a amenée à étudier la façon dont les acteurs élaborent des institutions les conduisant à être 
coopératifs. Depuis, de nombreux chercheurs ont progressé dans la connaissance des facteurs influençant les comportements de coopération : voir par exemple les travaux de Gatiso et al. (2015), qui testent les rôles de la démocratie et des sanctions sur la coopération ; d'Ohler and Billger (2014), qui montrent l'importance de prendre en compte les intérêts individuels plutôt que collectifs pour éviter la tragédie des communs ; et de Marshall (2004) qui souligne l'importance de prendre en compte des facteurs sociaux tels que la confiance, en plus des facteurs économiques généralement considérés pour l'élaboration de politiques publiques.

Ostrom (1990) a cherché à formuler des hypothèses sur les raisons pour lesquelles certains collectifs arrivaient à s'organiser alors que d'autres échouaient. Comparant un grand nombre de situations de gestion de ressources communes, et mobilisant diverses méthodes (analyses et méta-analyses de cas ; expérimentations et modélisations faisant appel à la théorie des jeux), elle a identifié huit principes de design institutionnel (design principles), qu'elle définit comme " éléments ou conditions essentiels au succès rencontré par ces institutions pour assurer la durabilité des ressources communes et obtenir la conformité de générations d'exploitants aux règles en vigueur » (Ostrom, 1990, p.90, notre traduction). Ces principes de design institutionnel sont encore utilisés comme référence pour diagnostiquer des cas de gouvernance des biens communs : voir par exemple Sarker et al. (2015) sur les pêcheries, qui s'intéressent au rôle de l'Etat pour les assurer, et Skurray (2015) qui les mobilise pour diagnostiquer l'échec de la gestion collective d'une nappe phréatique. 
1.3. : Hypothèses implicites de la modélisation des systèmes de ressources communes

Nous analysons à présent plus en détail une étude de cas emblématique ${ }^{2}$ d'Ostrom, celle des nappes phréatiques de Californie, afin de mettre en évidence certaines hypothèses qui circonscrivent le domaine d’application de son cadre théorique.

\subsubsection{Le cas des nappes phréatiques de Californie}

Ostrom précise d’emblée que les nappes phréatiques du sud de Los Angeles sont des biens d'une grande valeur dans cette région semi-aride, car elles fournissent de l'eau de bonne qualité et à faible coût comparé à celui de l’importation d'eau en provenance d'autres régions. De plus elles permettent de stocker l'eau en prévision de fortes demandes. L'auteur explique ensuite que la surexploitation de l'eau, entraînant un appauvrissement irréversible de la ressource et parfois des intrusions d'eau salée, menaçait l'ensemble des nappes phréatiques de la région jusqu'à ce que des changements institutionnels aient été initiés par les acteurs concernés. Elle qualifie la situation initiale de course au pompage (pumping race), dans laquelle les exploitants cherchaient à maximiser leur consommation en eau, et qui aurait pu mener à une tragédie des communs.

Ostrom montre qu'un changement s'est d'abord produit à la fin des années 1930 dans le bassin versant Raymond, le plus petit de la région, enclavé dans les terres, donc non soumis au risque d'infiltration d'eau salée. Environ 30 exploitants se partageaient la nappe phréatique en question. L’un des acteurs lança une procédure judiciaire contre les autres exploitants afin de déterminer la structure géologique du bassin et de savoir si le seuil d'exploitation soutenable de la nappe avait été dépassé ou non. L'étude hydrologique révélant que ce seuil était

\footnotetext{
${ }^{2}$ Ostrom a réalisé sa thèse sur ce cas et y consacre une place importante dans son livre de 1990.
} 
largement dépassé, le juge a recommandé aux exploitants de réduire leur niveau de pompage. Afin d'éviter une longue procédure judiciaire, les parties prenantes ont décidé de trouver un accord collectif, négocié sur la base du concept de prescription réciproque : ils se sont mis d'accord pour réduire chacun leur consommation d’un quart. Ils ont également créé un marché des droits d'eau pour permettre un rééquilibrage des quantités d'eau consommées. Ostrom souligne que les parties prenantes, en négociant leur propre accord, ont mis fin à la course au pompage plus rapidement et à moindre coût que ce qu'ils auraient pu obtenir par une procédure judiciaire.

\subsubsection{Analyse du cas et mise en évidence d'hypothèses implicites}

Nous pouvons tirer de ce cas quelques caractéristiques-clés des ressources communes étudiées par Ostrom. Tout d'abord, les ressources sont clairement identifiées: ici le système de ressources est la nappe phréatique, et l'eau potable est la seule ressource visée par les institutions décrites. La communauté impliquée dans les négociations n’est composée que des exploitants de la nappe, c'est à dire des acteurs qui s'approprient les unités de ressource. Cette communauté est donc plutôt bien identifiée et les attentes des acteurs qui la composent sont homogènes par rapport à la ressource. Le bien commun préexiste à l'action collective et son importance est reconnue par tous les exploitants. L'action collective est initiée lorsque les exploitants prennent conscience de la destruction de la ressource en eau. Le problème identifié est essentiellement de maintenir le stock d'eau dans les nappes phréatiques. Deux voies sont alors possibles: d'une part limiter la consommation d'unités de ressources, d'autre part maintenir, voire améliorer le système de ressources. Ostrom insiste davantage sur la première voie, décrivant en détail comment les exploitants ont réussi à trouver un accord sur les prescriptions mutuelles. Les actions de gestion sont essentiellement ciblées sur la 
limitation du prélèvement de ressources ; ce sont les arrangements pour arriver à l'appliquer collectivement qui font essentiellement l'objet de son analyse. En résumé, Ostrom estime que les ressources communes préexistent à l'action collective et sont clairement identifiées comme ayant de la valeur par les acteurs concernés.

Nous présentons à présent un cas empirique de gestion collective d'un agro-écosystème que nous avons étudié entre 2010 et 2013. Nous montrons tout d'abord que ce cas ne répond pas aux caractéristiques classiques des systèmes de ressources communes et que par conséquent il n'est pas possible de mobiliser le cadre théorique d'Ostrom comme tel. Cependant, en abordant ce cas avec une approche de conception, nous en tirons des résultats ouvrant de nouvelles pistes de recherche sur les biens communs.

\section{2 : L’exploration collective d'un agro-écosystème durable en Poitou-Charentes}

\subsection{Méthodologie et cadre théorique mobilisé}

Nous présentons une étude de cas longitudinale (Eisenhardt 1989) portant sur une initiative originale (Siggelkow 2007) impliquant un partenariat entre une coopérative agricole, la Coopérative Entente Agricole (CEA) et un centre de recherche en écologie, le Centre d’Etudes Biologiques de Chizé (CEBC) dans une région agricole de Poitou-Charentes, les plaines de Niort.

Les données ont été collectées entre 2010 et 2013 par le biais d'entretiens semi-directs, d'observation participante et d'analyse de documents ; nous avons pu ainsi trianguler les données de façon à mieux comprendre la situation (Yin 2009). Au total, 41 entretiens de $1 \mathrm{~h} 30$ à $2 \mathrm{~h}$ ont été menés en 2010-2011 auprès de chercheurs en écologie et en agronomie, d'agriculteurs, de techniciens de la CEA et de chambres d'agriculture, de représentants des 
services déconcentrés de l'Etat et d'organisations non gouvernementales, ainsi que de membres du conseil d'administration et de la direction de la CEA. Des notes détaillées ont été prises au cours de ces entretiens. Le premier auteur a passé six mois sur place en 2010 puis a assisté à quinze réunions organisées dans le cadre du projet de 2011 à 2013. Une courte phase de recherche-intervention (David, 2002) a conduit à la mise en place d'un atelier de conception innovante organisé suivant la méthode KCP (Knowledge-Concept-Proposal) (Hatchuel et al., 2009; Berthet et al., 2015). Cet atelier d’une journée a rassemblé en mai 2011 environ 30 participants (chercheurs, membres de la coopérative, techniciens agricoles et représentants d'institutions locales). L'analyse de documents a porté sur des publications scientifiques et travaux non publiés du CEBC, des documents fournis par la coopérative ainsi que des documents administratifs portant sur les politiques agricoles et environnementales appliquées sur le territoire étudié.

L'analyse des données a consisté à modéliser les raisonnements de conception menés par les acteurs en mobilisant les théories de la conception. Selon la théorie C-K du raisonnement de conception (Hatchuel and Weil 2009), le point de départ d'un tel raisonnement est une proposition indécidable (i.e., dont on ne peut dire si elle est vraie ou fausse), que l'on peut aussi qualifier de concept ou d'inconnu imaginable. Le raisonnement de conception consiste à simultanément étendre l'espace des connaissances en réunissant les connaissances disponibles et en en produisant de nouvelles, et étendre l'espace des concepts en dotant le concept initial de propriétés permettant de le spécifier (Hatchuel and Weil 2009). L'objectif d'un raisonnement de conception est d'obtenir une ou plusieurs propositions réalisables, mais aussi de structurer et enrichir l'espace des connaissances et d'explorer l'espace des concepts. Notre interprétation des raisonnements a été régulièrement confrontée aux données et partagée avec 
les acteurs (Glaser and Strauss, 1967) de façon à stabiliser notre compréhension de la situation.

\subsection{Une tentative d'auto-organisation dans un contexte différent de celui des CPR}

La région agricole étudiée, située en Poitou-Charentes dans les plaines de Niort, est dotée d'une biodiversité remarquable ; elle héberge 17 espèces d'oiseaux protégées aux niveaux nationaux et Européens. Parmi elles figure l'outarde canepetière (Tetrax tetrax), espèce patrimoniale dont la population en question est l'une des dernières en France. Cependant, depuis les années 1990, la biodiversité a beaucoup été altérée dans la région, et les effectifs d'outardes ont diminué de 90\% en 20 ans (Inchausti and Bretagnolle 2005). Selon les écologues, cette érosion de la biodiversité est directement liée à l'intensification agricole de la région (Bretagnolle et al. 2011), qui se traduit par une spécialisation dans les grandes cultures (céréales et oléo-protéagineux) et une industrialisation de l'élevage ovin et caprin incitant les éleveurs à acheter des aliments déshydratés. Cette dynamique a entraîné une diminution des cultures fourragères semi-pérennes, et plus généralement une homogénéisation des paysages ainsi qu'une forte augmentation de l'utilisation d'intrants chimiques.

A première vue, cette situation ressemble à une tragédie des communs, dans laquelle l’outarde canepetière (ou plus largement la biodiversité) serait le bien commun à préserver. En effet, la préservation de l'outarde (i) est menacée par les activités humaines; (ii) nécessite une coordination des acteurs, et (iii) correspond à un problème de passager clandestin ${ }^{3}$. Si l'on appliquait le cadre d'analyse d’Ostrom (1990), la plaine céréalière serait le système de ressources, et les outardes les ressources à préserver. L'objectif serait alors d'exclure ou de

\footnotetext{
${ }^{3}$ Le coût de la préservation de la biodiversité paraissant à un agriculteur supérieur au gain qu'il pourrait en attendre, il serait donc tenté de se comporter comme un passager clandestin et de ne pas y contribuer
} 
réduire toute pratique de gestion du système de ressources contribuant à une dégradation du « stock » d'outardes, en l'occurrence les pratiques liées à la production agricole intensive. C'était en quelque sorte la voie envisagée par les écologues et les naturalistes, mobilisés depuis 1996 pour mener des actions de conservation de l'outarde, incluant notamment l'achat de terres pour leur mise en jachère, et la mise en place de mesures agri-environnementales visant à limiter l’usage de pesticides ou à réintroduire des prairies.

Cependant, Ostrom (1990) précise que les ressources communes doivent avoir une valeur reconnue par l'ensemble de la communauté qui les gère ; or l'outarde canepetière n’a pas pour les agriculteurs une valeur comparable à celle du produit de leur activité. En outre, la communauté en charge de la préservation de l'outarde n’inclut pas les agriculteurs, ou seulement de façon très marginale, alors que ce sont eux qui ont le plus d'impact sur le système de ressources. La situation était d'ailleurs plutôt conflictuelle au milieu des années 2000 ; un groupe d'acteurs composés d'agriculteurs, de chasseurs et de forestiers ayant bloqué pendant plusieurs années les négociations autour de la mise en place et de la gestion d'une zone de conservation Natura 2000. Par conséquent, il était difficile d'envisager la mise en place d'une action auto-organisée impliquant l'ensemble de la communauté d'acteurs autour de la préservation de l'outarde canepetière.

Pourtant, lorsqu'une coopérative agricole locale, la CEA a initié fin 2010 un partenariat avec le CEBC, l'ambition était d'explorer une nouvelle voie par rapport à celle de la régulation publique basée sur les instruments d'incitation économique individuelle, celle de l'autoorganisation. La coopérative sollicitait les écologues pour leur expertise sur la biodiversité, afin d'identifier des solutions permettant de rendre l'agriculture plus durable au plan environnemental. Les écologues ont d'emblée proposé à la coopérative de développer une 
filière courte de luzerne, en vue d'augmenter la production de cette culture intéressante au plan écologique et agronomique. Il s'agissait de développer un modèle d'affaire viable économiquement, ne nécessitant pas de subventions publiques.

Le choix de la luzerne était motivé par plusieurs raisons : au plan écologique et environnemental, la luzerne est un lieu de refuge et d'alimentation pour les outardes, ainsi que pour des insectes dont elles se nourrissent ; de plus, cette culture ne nécessitant peu ou pas de pesticides, est intéressante pour la biodiversité et la qualité de l'eau. Au plan agricole et agronomique, c'est un fourrage de qualité pour l'élevage bovin et caprin, qui peut être rentabilisé, contrairement à la jachère ; de plus, cette culture améliore la structure et la fertilité des sols. On pouvait donc penser que le nouveau bien commun à gérer devenait la luzerne, puisque les agriculteurs, aussi bien que les naturalistes et les citoyens, avaient intérêt à maintenir voire augmenter les surfaces en luzerne dans la plaine céréalière.

Cependant, le projet de filière luzerne s’est rapidement heurté à plusieurs difficultés : les agriculteurs, en particulier les céréaliers, n’étaient pas favorables à l’idée d’augmenter les surfaces en luzerne, alors que cette production était moins rentable à l'année que les grandes cultures et qu'il n’existait pas réellement de marché de foin de luzerne. Par ailleurs, pour les agriculteurs, produire de la luzerne n’avait de sens que si l'on en maximisait le rendement à la parcelle, ce qui impliquait des pratiques de gestion intensives (usage d'herbicides et d'engrais ; irrigation si possible ; 3 à 4 fauches par an ; retournement au bout de 3 ou 4 ans) ; alors que pour les écologues, une luzerne intéressante au plan écologique était la moins perturbée possible (pas d'intrants ni d'irrigation ; au plus 3 fauches par an ; luzerne laissée en place au moins 5 ans). 
On pouvait donc se poser la question : de quelle luzerne parlait-on, et a fortiori de quel bien commun parlait-on? Tant que l'on faisait l'hypothèse que la luzerne était donnée et son identité connue, les parties prenantes du projet ne semblaient pas pouvoir se mettre d'accord. C’est à partir de ce constat que les porteurs du projet de filière luzerne se sont lancés dans une démarche de conception innovante, qui, précisément, ne considérait pas la luzerne comme un bien commun donné, mais comme étant à concevoir.

\subsection{Une démarche d'exploration pour surmonter un blocage de l'action collective}

L'atelier de conception a démarré par le partage d'une diversité de connaissances (agronomiques, zootechniques, écologiques et socio-économiques). L’accent a cependant été mis sur l'écologie qui permettait de bien comprendre le fonctionnement du système de ressources, l’agro-écosystème. Selon les écologues du CEBC, les « prairies » ou cultures semi-pérennes, telles que la luzerne, constituent en région céréalière des zones de reproduction et de refuge pour de nombreuses espèces, en particulier pour les insectes dont se nourrit l'outarde canepetière. Or d'après la théorie des métapopulations (Hanski 1999), si l'agro-écosystème est représenté comme une mosaïque paysagère composée d'espaces de haute valeur écologique (les prairies) et d'espaces de faible valeur écologique (les grandes cultures), la distance entre les espaces de haute valeur écologique va conditionner la capacité des populations d’insectes à recoloniser les espaces dans lesquels ils ont été ponctuellement détruits (ici, les grandes cultures). Ainsi, le maintien d'une proportion minimale de prairies dans la plaine cultivée apparaissait comme une propriété-clé de l’agro-écosystème conciliant agriculture et biodiversité. Les écologues ont évalué à 10\% la proportion minimale de prairies à maintenir, de façon suffisamment répartie dans l'espace pour que les distances entre prairies permettent une recolonisation du milieu par les insectes. 
L’approche par la conception proposée dans l'atelier a mis en évidence que si l'écologie indiquait une voie à suivre, celle de maintenir une mosaïque paysagère composée de minimum $10 \%$ de prairies, elle ne présumait pas a priori de la configuration définitive du paysage. On pouvait faire l'hypothèse qu'il existait de nombreuses formes possibles de mosaïques paysagères, dont toutes n’étaient pas connues. La proposition " mosaïque paysagère composée de minimum 10\% de prairies » constituait un inconnu imaginable (Hatchuel et Weil 2009), donc le point de départ d'un processus de conception. En effet, la mosaïque paysagère peut être conçue en introduisant des milieux semi-pérennes (luzerne), pérennes (prairies permanentes, jachères) ou en introduisant de la culture biologique et sans labour. Par ailleurs, les valeurs d'une mosaïque paysagère ne sont pas non plus toutes connues à l'avance : selon sa configuration, un paysage peut assurer la préservation d'une espèce menacée, améliorer la qualité de l’eau, ou encore se voir attribuer une valeur esthétique supérieure à celle d’un paysage de monoculture.

retenue, il est possible de jouer sur sa distribution pour favoriser le repeuplement en insectes, ou encore sur les dates de fauches pour favoriser les pollinisateurs en maintenant dans le paysage des parcelles en floraison (Requier et al. 2015). L'atelier de conception collective et le projet de recherche-action qui a suivi ont permis d'avancer dans cette exploration d'un inconnu commun. 
3. L’inconnu commun : initier une action collective en l'absence de bien commun

\subsection{L’agro-écosystème, un système plus complexe que les archétypes d’Ostrom}

Les agro-écosystèmes ne semblent pas répondre aux critères indiqués par Ostrom pour caractériser des systèmes de ressources communes (Common Pool Resources). En effet, le système de ressources est difficile à définir, notamment parce que les ressources à gérer en commun sont multiples, leurs flux peuvent être antagonistes et il n'existe pas de consensus a priori sur ceux à maximiser. Les valeurs des ressources sont souvent perçues différemment par les parties prenantes, ce qui peut créer des situations de conflits. Les limites physiques d'un agro-écosystème ne peuvent pas être définies de manière générale ; elles peuvent l’être en fonction des flux ciblés (ex. bassin versant si l'on cible la qualité de l'eau), ou encore en fonction de l'autorité de gestion que l'on prend en compte (ex. territoire de la coopérative). De plus, la communauté qui pourrait être chargée de concevoir les institutions adéquates est difficile à identifier puisque les acteurs dépendants de l'agro-écosystème ont généralement des intérêts hétérogènes voire divergents.

Par ailleurs, la méthode par essais-erreurs ou la résolution de problème proposées par Ostrom (1990) ne semblent pas adaptées à la conception de nouvelles méthodes de gestion de l'agroécosystème : d’une part, la connaissance fait défaut en ce qui concerne son fonctionnement, et il est difficile de mettre en place des expérimentations pour étudier des interactions aussi complexes, concernant des échelles spatiales et temporelles parfois très larges. D’autre part l’identité-même de l'agro-écosystème n’est pas stable au cours du temps. De nouvelles valeurs ou utilisations des produits de l'agro-écosystème sont sans cesse inventées ; on peut citer par exemple les innovations concernant l'utilisation du chanvre à des matériaux de construction (Le Masson et al. 2012a) ou encore les biocarburants. Notre analyse montre que 
dans le cas des agro-écosystèmes, il peut être fructueux de passer d'une logique de préservation de biens communs à une logique d'exploration d'un inconnu commun.

\subsection{Identifier des inconnus communs pour stimuler les capacités d'innovation}

Le Masson et Weil (2014) distinguent quatre types d’inconnus : (i) les inconnus réalisables, i.e., ceux que les entreprises sont facilement capables d'explorer ; (ii) les inconnus accessibles (à un effort de conception près) qui peuvent se recouper entre les entreprises, ce qui limite leur capacité à se différencier les unes des autres ; (iii) les inconnus imaginables qui permettent d'ouvrir l'exploration de voies nouvelles ; et (iv) les inconnus inaccessibles. Les auteurs soulignent que déplacer la frontière des inconnus imaginables est une manière de traiter l’inconnu inaccessible, donc d'accéder à l’innovation.

Les inconnus imaginables générés pour stimuler les capacités d’innovation des entreprises sont qualifiés par les auteurs d' « inconnus communs », un qualificatif qui met en évidence l'intérêt que peut avoir une communauté d'entreprises (ou un écosystème d’innovation) à les maintenir en commun et à lutter contre leur appropriation par une ou plusieurs entreprises, afin d'éviter l'épuisement de la capacité d’innovation de l'ensemble de la communauté. Le Masson et Weil (2014) indiquent en effet que l'appropriation prématurée de l'inconnu commun peut susciter des conflits si l'exploration n’a pas été suffisamment poussée pour prendre en compte les intérêts de tous les acteurs, ou encore peut susciter des effets de fixation si celui qui se l’approprie est « fixé ».

Nous avons repris la notion d' « inconnu commun » introduite par Le Masson et Weil (2014) pour désigner une proposition qui permet d'initier un processus de conception impliquant une 
diversité de parties prenantes, sans préjuger de ce qui pourrait constituer pour elles un bien commun.

\subsection{Mobiliser les connaissances en écologie pour qualifier l'inconnu commun}

Dans le cas étudié, c’est à partir des connaissances en écologie qu'a pu être identifié l'inconnu commun «mosaïque paysagère composée d'au moins 10\% de prairies ». Par sa capacité de modélisation, l'écologie a permis de mettre en évidence une propriété clé de l'agroécosystème à préserver, constituant en quelque sorte une spécification initiale ou un brief de départ du processus de conception, et ouvrant un champ d'exploration pouvant guider une diversité d'acteurs. La démarche de conception innovante est partie des connaissances en écologie pour qualifier un inconnu, puis faire participer les différentes parties prenantes à l'exploration de cet inconnu. Une telle démarche ouvre des perspectives nouvelles, qui ne consistent non pas à préserver un système donné, mais à explorer de nouveaux systèmes, tout en respectant une propriété écologique clé. Ainsi, il ne s’agit pas d’une situation classique d'auto-organisation pour la préservation de biens communs, mais d'une exploration de ce qui peut être fait en commun : à la fois en termes de modes de gestion de la mosaïque paysagère et en termes d'exploration des valeurs potentielles générées par telle ou telle configuration. Les acteurs sont invités à échanger leurs intérêts, connaissances et critères d'évaluation respectifs, mais aussi à réfléchir ensemble à de nouvelles façons de gérer l'agro-écosystème. 


\subsection{L’inconnu commun pour surmonter un blocage de l'action collective}

Le cas étudié souligne l’intérêt de générer des inconnus imaginables permettant des processus de conception collectifs, afin de mobiliser une communauté d’acteurs hétérogènes qui ne sont pas forcément enclins à coopérer initialement. L’hypothèse sous-jacente est qu'en incitant les acteurs à explorer collectivement des projets nouveaux, la coopération sera rendue plus facile que dans un processus de négociation classique, où chaque partie défend ses intérêts et risque de perdre des acquis à l'issue du processus.

A titre d'exemple, afin d'illustrer en quoi un raisonnement de conception peut aider à sortir d'une impasse, si l’on fait l’hypothèse que les agriculteurs attribuent à la luzerne la propriété P1 « sans adventices » (pour des raisons de qualité du fourrage), et les écologues lui attribuent la propriété P2 « sans herbicides » (car ces derniers nuisent à la biodiversité), a priori, ces deux propriétés semblent incompatibles. Selon la théorie C-K, la luzerne devient un inconnu à partir du moment où on lui attribue un ensemble de propriétés initialement incompatibles, et où l'on ne considère pas que les concilier est " impossible », mais " indécidable ». La proposition « une luzerne dans adventices et sans herbicides » peut alors être considérée comme un inconnu commun. Un certain nombre de recherches ont instruit la possibilité d'une gestion des adventices dans les luzernes sans utiliser d'herbicides (Meiss et al. 2008). Elles montrent que si une parcelle est envahie par les adventices en première année, la gestion des coupes de luzerne peut aider la culture à reprendre le dessus pour les années suivantes. Ces nouvelles connaissances permettent d'identifier une propriété P3 relative aux rythmes de fauches, qui rend P1 et P2 compatibles. 


\section{Discussion :}

4.1. Des biens communs aux inconnus communs : mise en évidence de nouveaux enjeux de gestion

Dans les cas étudiés par Ostrom, les ressources sont considérées comme données et les actions de gestion prises en compte se limitent essentiellement à réguler leur prélèvement. Comme le souligne Fournier (2013), Ostrom s’est surtout intéressée aux problématiques d’allocation et de non-appropriation des biens, sujets qui mobilisent encore la plupart des travaux sur les biens communs. Notre recherche suggère que, dans des cas plus complexes tels que les agroécosystèmes, il peut être fructueux de passer d'une logique de préservation de biens communs à une logique d'exploration d'inconnus communs. L'exploration va porter simultanément sur un ensemble de dimensions liées entre elles : l’agro-écosystème, les valeurs associées, le collectif de conception, les actions de gestion à mettre en place, les règles de gouvernance et le modèle économique pertinents. Un tel processus soulève divers enjeux de gestion qui semblent aujourd'hui encore sous-estimés dans la littérature sur les biens communs. Nous en identifions quatre et montrons que l'identification et l'exploration collective d'inconnus communs semblent constituer une approche pertinente pour y répondre.

Tout d'abord, nous avons montré que dans les agro-écosystèmes, il n’existait pas a priori de consensus sur ce qui peut être géré en commun. La multiplicité des objectifs de gestion possibles et les divergences d’intérêts peuvent créer des situations conflictuelles entre les parties prenantes de l'agro-écosystème. Afin de surmonter les éventuels conflits, tout au moins les situations de blocage de l'innovation, le fait d'engager un processus collectif de conception à partir de l'identification d'un (ou plusieurs) inconnu(s) commun(s) incite des acteurs aux intérêts divergents à explorer ensemble des solutions « désirables ». 
Un deuxième enjeu de gestion pour améliorer la durabilité des agro-écosystèmes est d'inciter les acteurs à explorer des voies innovantes, donc à surmonter les verrouillages (Liebowitz and Margolis 1995) et les effets de fixation cognitifs (Agogué et al. 2014). D’après Le Masson et Weil (2014), les inconnus communs, s’ils sont provoquants et stimulants, peuvent contribuer à surmonter ces effets de fixation. Pour cela, ces auteurs soulignent qu’il est nécessaire de repérer et évaluer les voies de rupture et de mutualiser l’identification de lacunes de connaissances. L’élaboration et la diffusion de méthodes de conception collective peuvent y aider. Dans le cas empirique, nous avons appliqué la méthode KCP (Hatchuel et al. 2009), mais il existe d'autres méthodes de conception collective qui peuvent être mobilisées en contexte agricole (Berthet et al. 2015). L’élaboration de règles d'action collectives permettant, voire stimulant, l’innovation, est également une piste complémentaire. Le Masson et al. (2012b) mettent en évidence de l'existence de ce qu’ils nomment « règles de déverrouillage » (unlocking rules) pour susciter l’innovation à l'échelle des régimes sociotechniques, souvent caractérisés par des logiques de « verrouillage ». Enfin, l’exploration de l’inconnu commun va nécessiter des savoirs dans des disciplines multiples (écologie, agronomie, sciences économiques et sociales) et qui peuvent être de natures variées (scientifique, empirique, technique...). L'identification d'experts pertinents à impliquer dans le processus de conception peut également conditionner sa réussite.

Un troisième enjeu de gestion, est lié au fait que les acteurs des agro-écosystèmes, notamment les agriculteurs, prennent généralement leurs décisions de façon indépendante. Or certaines problématiques environnementales nécessitent une coordination des actions à l'échelle du territoire (Goldman et al. 2007). L'identification et l'exploration d'inconnus communs peut aider à rendre visibles les interdépendances entre acteurs, à la fois concernant les actions de gestion (e.g., coordonner les dates de fauche de la luzerne pour favoriser les pollinisateurs) et 
leurs bénéfices potentiels (e.g., reconnaître l'enjeu pour les apiculteurs des actions portées par les agriculteurs), et ainsi faciliter la coordination des leurs actions.

Enfin un quatrième enjeu identifié est lié au fait que l'agro-écosystème est dynamique et soumis à de nombreuses perturbations, dont certaines sont influencées par le processus de conception collective. Il est par conséquent important d'assurer un suivi à long terme de la trajectoire de l'agro-écosystème, mais aussi de maintenir dans le temps la possibilité de relancer des phases d'exploration collective. La régénération progressive des inconnus communs peut aider les acteurs à agir dans un contexte dynamique tel que celui des agroécosystèmes.

\subsection{Une contribution à la littérature visant à dénaturaliser les biens communs}

Cet article contribue à un ensemble de travaux de recherche émergents qui discutent l'approche naturaliste des biens communs et soulignent les enjeux de gestion aujourd'hui peu pris en compte dans la littérature. On peut citer par exemple les travaux de Labatut (2009), qui s'est intéressée aux processus d'apprentissage ayant permis la co-construction des collectifs et des objets de gestion des biens communs (en l'occurrence des races animales locales), constituant ce qu'elle qualifie de « dispositifs coopératifs ». Les travaux d'Hannachi et al. (2010), eux, considèrent le territoire agricole comme un bien commun résultant d'une construction sociale. Selon ces auteurs, si les acteurs d'un territoire en identifient des propriétés pouvant leur procurer un avantage compétitif, alors ils auront tendance à les préserver collectivement et à éviter les comportements déviants. De même, selon Fournier (2013), les biens communs ne sont pas un ensemble fini de ressources, mais des processus sociaux de production et d'organisation. Par conséquent, cet auteur souligne l'importance de ne pas penser seulement l'allocation des ressources, mais aussi les problématiques 
organisationnelles relatives à l'usage et à la production des biens communs. Elle qualifie ce processus de « commoning », suggérant, à l'instar de Linebaugh (2007) et de Dardot et Laval (2015), les communs devraient être davantage considérés comme un verbe traduisant une action plutôt que comme un nom. Partageant ces points de vue, cet article présente l'identification et l'exploration d'inconnus communs comme une méthode grâce à laquelle des acteurs pourraient initier une démarche de conception de biens communs. Une telle méthode pourrait initier, à un niveau local, la conception d'un champ d'innovation évoquée par Aggeri (2011), qui « polarise les efforts et les capacités d'autres acteurs en vue d'élaborer un nouveau bien commun » et qui permet d'explorer de nouveaux potentiels de valeur d'un agro-écosystème.

\section{Conclusion}

Cette recherche s’est appuyée sur une analyse détaillée d'un cas étudié par Ostrom et d'un cas empirique de gestion collective d'un agro-écosystème, pour mettre en évidence certaines hypothèses implicites de la littérature classique sur les biens communs. Elle révèle que ces hypothèses excluent un certain nombre de situations dans lesquelles une action collective auto-organisée pourrait être une bonne alternative à la régulation publique, alors qu’il n’existe pas a priori de biens communs identifiés comme tels. La notion d'inconnu commun permet d'initier une action collective en l'absence de biens communs ; par conséquent, elle désigne une classe d'actions plus large que celle des ressources communes modélisées par Ostrom, et dans lesquelles les enjeux de gestion peuvent être beaucoup plus complexes que la limitation du prélèvement des ressources. Afin d'enrichir cette analyse, il serait intéressant d'étudier comment, dans d'autres contextes que celui de l'agriculture, la notion d'inconnu commun 
permettrait d'interpréter des situations dans lesquelles des acteurs parviennent à initier des formes innovantes d'action collective alors qu'ils ne sont pas liés initialement par des biens communs.

\section{Remerciements}

Les auteurs remercient Vincent Bretagnolle, Egizio Valceschini, Pascal Le Masson, Mourad Hannachi et Emmanuel Raynaud pour leur contribution à la réflexion sur ce sujet. Cette recherche a été financée par l'INRA et par le programme Agreenskills+, financé par le 7e Programme Cadre Européen dans le cadre du contrat No. FP7-609398.

\section{Bibliographie}

Aggeri F. (2011), « Le développement durable comme champ d'innovation », Revue française de gestion, vol.6, p.87-106.

Agogué M., Kazakçi A., Hatchuel A., Le Masson P., Weil B., Poirel N., Cassotti M. (2014), " The impact of type of examples on originality: Explaining fixation and stimulation effects ", The Journal of Creative Behavior, vol.48, $n^{\circ} 1$, p.1-12.

Berthet E.T., Barnaud C., Girard N., Labatut J., Martin G. (2015), « How to foster agroecological innovations? A comparison of participatory design methods », Journal of Environmental Planning and Management, vol.59, n², p. 280-301.

Billaudot B. (2013), «Qu'est qu'un bien commun? », Revue de l'organisation responsable, vol.7, n², p.31-45.

Bretagnolle V., Gauffre B., Meiss H., Badenhausser I. (2011), « The role of grassland areas within arable cropping systems for the conservation of biodiversity at the regional level ", in Grassland Productivity and Ecosystem Services, coordonné par G. Lemaire, J. A. Hodgson and A. Chabbi: p.251-260.

Dardot P., Laval C. (2015), Commun: essai sur la révolution au XXIe siècle, La Découverte, Paris. 
David A. (2002), "Intervention methodologies in management research », EURAM Conference, Stockholm, p. 9-11.

Eisenhardt K.M. (1989) « Building theories from case study research », Acad. Manage. Rev., vol.14, ${ }^{\circ} 4$, p.532-550.

Fournier V. (2013) "Commoning: on the social organisation of the commons", M@n@gement, vol.16,n4, p.433-453.

Gatiso T.T., Vollan B., Nuppenau E.A. (2015), « Resource scarcity and democratic elections in commons dilemmas: An experiment on forest use in Ethiopia », Ecological Economics, vol.114, p.199-207.

Glaser B., Strauss A. (1967), The Discovery Grounded Theory: Strategies for Qualitative Inquiry, Aldin, Chicago.

Goldman R.L., Thompson B.H., Daily G.C. (2007), « Institutional incentives for managing the landscape: inducing cooperation for the production of ecosystem services ", Ecological Economics, vol.64, n², p.333-343.

Hannachi M., Coleno F.C., Assens C. (2010), « La collaboration entre concurrents pour gérer le bien commun : Le cas des entreprises de collecte et de stockage de céréales d'Alsace », Gérer et Comprendre. Les Annales des Mines, p.16-25.

Hanski I. (1999) « Habitat connectivity, habitat continuity, and metapopulations in dynamic landscapes », Oikos, vol.87, n², p.209-219.

Hardin G. (1968), « The tragedy of the commons », Science, vol.162, p.1243-1248.

Hatchuel A., Weil B. (2009), «C-K design theory: an advanced formulation », Research in Engineering Design, vol.19, p.181-192.

Hatchuel A., Le Masson P., Weil B. (2009), « Design theory and collective creativity: a theoretical framework to evaluate KCP Process », Int. Conf. on Engineering Design, ICED’09, Stanford, CA, USA.

Inchausti P., Bretagnolle V. (2005), «Predicting short-term extinction risk for the declining Little Bustard (Tetrax tetrax) in intensive agricultural habitats », Biological Conservation, vol.122, n³, p.375-384.

Labatut J. (2009), Gérer des biens communs: processus de conception et régimes de coopération dans la gestion des ressources génétiques animales, École Nationale Supérieure des Mines de Paris.

Le Masson P., Aggeri F., Barbier M., Caron, P. (2012a), "The sustainable fibres of generative expectation management: The "building with hemp" case study ", in System Innovations, Knowledge Regimes, and Design Practices towards Transitions for Sustainable Agriculture, coordonné par M. Barbier and B. Elzen. Paris, INRA Editions: p.226-251. 
Le Masson P., Weil B., Hatchuel, A., Cogez, P. (2012b), « Why are they not locked in waiting games? Unlocking rules and the ecology of concepts in the semiconductor industry », Technology Analysis \& Strategic Management, vol.24, nº6, p.617-630.

Le Masson P., Weil B. (2014) «Réinventer l'entreprise: la gestion collégiale des inconnus communs non appropriables ", in L'entreprise point aveugle du savoir, coordonné par Segrestin, B., Roger, B., Vernac, S., Ed. Sciences Humaines.

Liebowitz S. J., Margolis, S. E. (1995), « Path dependence, lock-in, and history », Journal of Law, Economics \& Organization: p.205-226.

Linebaugh P. (2008), The Magna Carta manifesto: liberties and commons for all, Univ of California Press.

Marshall G. R. (2004), « Farmers cooperating in the commons? A study of collective action in salinity management », Ecological economics, vol.51, n³, p.271-286.

Meiss H., Munier-Jolain N., Henriot F., Caneill J. (2008), «Effects of biomass, age and functional traits on regrowth of arable weeds after cutting ", Journal of Plant Diseases and Protection, vol.21, p.493-499.

Ohler A. M., Billger S.M. (2014), «Does environmental concern change the tragedy of the commons? Factors affecting energy saving behaviors and electricity usage », Ecological Economics, vol.107, p.1-12.

Olson M. (1965), The Logic of Collective Action. Cambridge, MA, Harvard University Press.

Ostrom E., (1990) Governing the Commons. The Evolution of Institutions for Collective Action. Cambridge University Press.

Requier F., Odoux J.-F., Tamic T., Moreau N., Henry M., Decourtye A., Bretagnolle, V. (2015), " Honey bee diet in intensive farmland habitats reveals an unexpectedly high flower richness and a major role of weeds », Ecological Applications, vol.25, p.881-890.

Sarker A., Ikeda T., Abe T., Inoue, K. (2015), «Design principles for managing coastal fisheries commons in present-day Japan », Ecological Economics, vol.117, p.32-38.

Siggelkow N. (2007) «Persuasion with case studies », Acad. Manage. J., vol.50, n²1, p.2024.

Skurray J. H. (2015), " The scope for collective action in a large groundwater basin: an institutional analysis of aquifer governance in Western Australia », Ecological Economics, vol.114, p.128-140.

Yin R.K. (2009), Case study research. Design and methods. Fourth edition. Applied social research methods series, v.5. 